\title{
The BP Tau disk: A missing link between Class II and III objects?
}

\author{
A. Dutrey ${ }^{1}$, S. Guilloteau ${ }^{2}$, and M. Simon ${ }^{3}$ \\ ${ }^{1}$ Laboratoire d'Astrophysique de l'Observatoire de Grenoble, BP 53, 38041 Grenoble Cedex 9, France \\ 2 Institut de Radio Astronomie Millimétrique, 300 rue de la Piscine, 38406 Saint Martin d'Hères, France \\ 3 Dept. of Physics and Astronomy, State Univ. of New York, Stony Brook, NY 11794-3800, USA
}

Received 9 August 2002 / Accepted 30 January 2003

\begin{abstract}
We present new single-dish (30-m) observations and an improved analysis of our earlier interferometric observations (Simon et al. 2000) of BP Tau^. Our analysis yields a detailed description of the properties of the circumstellar disk. The disk is small (outer radius $120 \mathrm{AU}$ ) and marginally optically thick in the ${ }^{12} \mathrm{CO} J=2 \rightarrow 1$ line. It is also relatively hot, about $50 \mathrm{~K}$ at $100 \mathrm{AU}$. The mm continuum emission is consistent with a thermal emission from circumstellar dust with an emissivity index $\beta \simeq 0.7$, and a disk mass about $1.2 \times 10^{-3} M_{\odot}$. The anomalously low ${ }^{12} \mathrm{CO}$ to dust emission ratio implies that either $\mathrm{CO}$ is depleted by a factor of the order of 150 with respect to $\mathrm{H}_{2}$, or has a very low gas to dust ratio, or highly anomalous dust properties. The disk exhibits direct evidence of Keplerian rotation, but because of a combination of insufficient resolution and optically thin CO emission, the mass derived for the star remains inaccurate (between 0.6 and $1.3 M_{\odot}$ for $140 \mathrm{pc}$ ). The unusual properties of the circumstellar disk suggest that BP Tau may be a transient object in the process of clearing its disk.
\end{abstract}

Key words. stars: circumstellar matter - stars: pre-main sequence - radio lines: stars

\section{Introduction}

CO line observations of low-mass Pre-Main-Sequence (PMS) stars in Taurus-Auriga ( $140 \mathrm{pc}$, Kenyon et al. 1994) provide strong evidence that many of them are surrounded by large $\left(R_{\text {out }} \sim 200-800 \mathrm{AU}\right)$ Keplerian disks (GM Aur: Koerner et al. 1993; GG Tau: Dutrey et al. 1994; MWC 480: Mannings et al. 1997). On the other hand, recent submillimeter, NIR and optical observations of nearby young stars that have already reached the Zero-Age-Main-Sequence (ZAMS) reveal that stars such as $\beta$ Pic are surrounded by optically thin dust disks ( $\beta$ Pic: Mouillet et al. 1997; Vega: Holland et al. 1998, HR 4796: Schneider et al. 1999). These disks, unlike the ones surrounding T Tauri stars, do not contain a significant amount of gas (Lagrange et al. 1995) and dust (Chini et al. 1991). They are thought to be second generation disks, associated with young stars that have already dissipated the gas and dust surviving from the parent molecular cloud, possibly to form planets. In these "debris disks", the dust is believed to come from the collision of larger bodies while most of the gas would be released from falling and evaporating bodies (FEB) similar to comets, e.g. $\beta$ Pic (Vidal-Madjar et al. 1998). Astronomers have not been successful in identifying stars that are dissipating their primary gas and dust disks in transition between having

Send offprint requests to: A. Dutrey,

e-mail: Anne.Dutrey@obs.ujf-grenoble.fr

* Based on observations carried out with the IRAM Plateau de Bure Interferometer. IRAM is supported by INSU/CNRS (France), MPG (Germany) and IGN (Spain). primary and secondary disks. T Tauri stars older than $10^{7}$ years (usually Weak-line T Tauri stars and Naked T Tauri stars) do not present $\mathrm{mm}$ emission in either $\mathrm{CO}$ or in the submillimeter continuum (Osterloh \& Beckwith 1995; Duvert et al. 2000).

BP Tau is a prominent "classic" T Tauri (CTT) star (e.g. Herbig \& Bell 1988). It experiences high mass accretion $\dot{M} \sim$ $3 \times 10^{-8} M_{\odot} \mathrm{yr}^{-1}$ from its circumstellar disk, which produces its strong excess emission in the ultraviolet, visible, and near infrared (Gullbring et al. 1998, 2000). It is very young; Gullbring et al.(1998) estimate $6 \times 10^{5} \mathrm{yr}$ for its age. Yet, despite these strong CTT characteristics, we found that its ${ }^{12} \mathrm{CO} J=2 \rightarrow 1$ line emission is unexpectedly weak, apparently because it is optically thin (Simon et al. 2000, hereafter SDG). Realizing that BP Tau could represent an object that is dissipating its disk at a very young age, we undertook further analysis of our millimeter wave observations of this star. We obtained new singledish CO observations. Using improved phase calibration techniques, we carried out a recalibration of our 1998-99 data and also were able to add data originally unusable. Our new analysis enables us to derive properties of BP Tau's circumstellar disk. We report our results here.

\section{Observations}

\subsection{PdBI data}

The observations used 5 antennas and were carried out in winter 1998/1999 in D, C2 and B1 configurations. Baselines up to $300 \mathrm{~m}$ provided $1.05 \times 0.90^{\prime \prime}$ resolution at $\mathrm{PA} 37^{\circ}$ for 


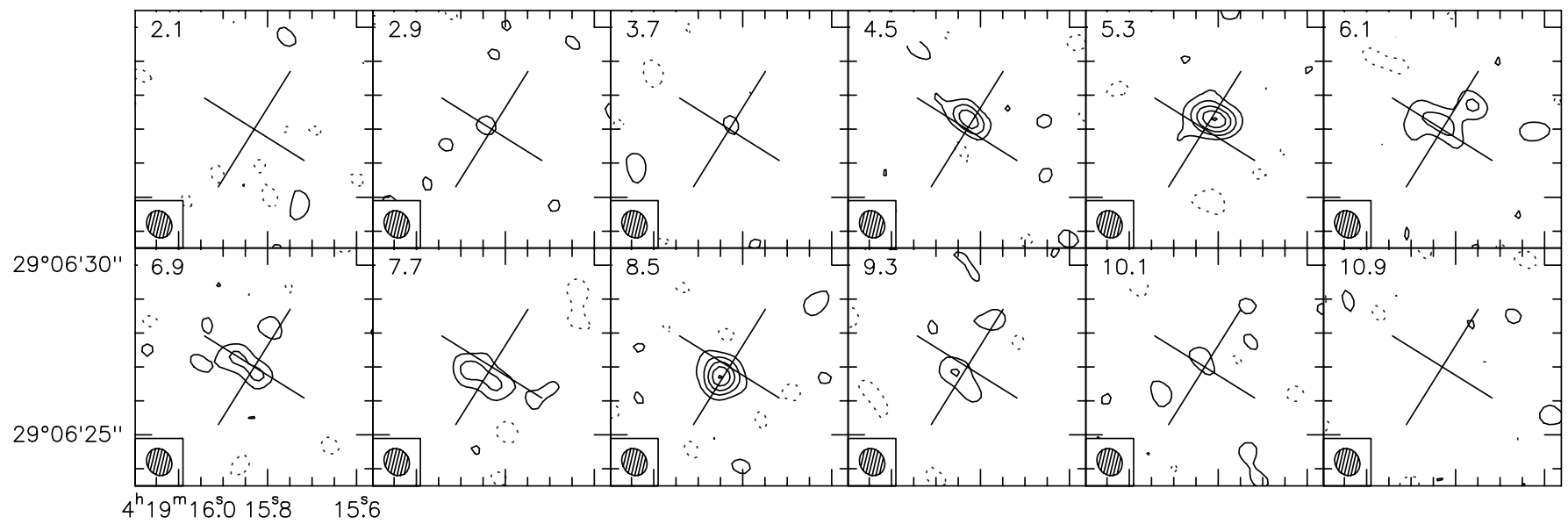

Fig. 1. Channel maps of the BP Tau ${ }^{12} \mathrm{CO} J=2 \rightarrow 1$ emission, at $0.8 \mathrm{~km} \mathrm{~s}^{-1}$ resolution, with the LSR velocity (in $\mathrm{km} \mathrm{s}^{-1}$ ) indicated in the upper left corner of each panel. The continuum emission has been subtracted. Contour levels are in steps of $25 \mathrm{mJy} /$ beam, or $0.61 \mathrm{~K}$ (approximately $2 \sigma)$, with the zero level omitted. The cross indicates the orientation of the disk.

the $1.3 \mathrm{~mm}$ continuum data. The total on source integration time is $\sim 20$ hours. We observed simultaneously at $89.2 \mathrm{GHz}$ $\left(\mathrm{HCO}^{+} J=1 \rightarrow 0\right)$ and $230.5 \mathrm{GHz}\left({ }^{12} \mathrm{CO} J=2 \rightarrow 1\right)$. At $1.3 \mathrm{~mm}$, the tuning was double-side-band (DSB) while at $3.4 \mathrm{~mm}$, the tuning was purely single-side band (LSB). The backend was a correlator with one band of $10 \mathrm{MHz}$ (spectral resolution $0.23 \mathrm{~km} \mathrm{~s}^{-1}$ ) centered on the $\mathrm{HCO}^{+} J=1 \rightarrow 0$ line, one band of $20 \mathrm{MHz}\left(0.18 \mathrm{~km} \mathrm{~s}^{-1}\right.$ resolution) centered on the ${ }^{12} \mathrm{CO} \mathrm{J}=$ $2 \rightarrow 1$ line, and 2 bands of $160 \mathrm{MHz}$ for the $1.3 \mathrm{~mm}$ and $3.4 \mathrm{~mm}$ continuum, respectively. The phase and flux calibrators were $0415+379$ and $0528+134$. The rms phase noise was $8^{\circ}$ to $25^{\circ}$ and $15^{\circ}$ to $50^{\circ}$ at $3.4 \mathrm{~mm}$ and $1.3 \mathrm{~mm}$, respectively, which introduced position errors of $<0.1^{\prime \prime}$. The estimated seeing is about $0.3^{\prime \prime}$.

Between 1999 and 2000, we improved at IRAM the method to calibrate the $1.3 \mathrm{~mm}$ data (details can be found in the manuals given at http://iram. fr/PDBI/bure.html). The long baseline observations were re-calibrated accordingly, and some data originally unusable were added. Because the disk is so small, small phase calibration errors and/or seeing effects can results in systematic effects making the disk appear more faceon. Amplitude errors can also have a similar effect. These effects are partially removed by the new calibration process.

We used the GILDAS software package to reduce the data. Images were produced in natural weighting of the visibilities for both the continuum and line maps.

We detected the CO $J=2 \rightarrow 1$ line emission and $1.3 \mathrm{~mm}$ and $3.4 \mathrm{~mm}$ continuum emission in BP Tau. Figure 1 presents channel maps of the $\mathrm{CO} J=2 \rightarrow 1$, with the velocity gradient map. Compared to SDG, the figure appears qualitatively similar. Figure 2 shows a comparison of the integrated $\mathrm{CO} J=$ $2 \rightarrow 1$ map and the $1.3 \mathrm{~mm}$ map. Continuum emission was subtracted to produce the $\mathrm{CO}$ maps presented in Figs. 1, 2 and the integrated flux given in Table 1. Flux density measurements and formal deconvolved Gaussian source sizes are given in Table 1 for the dust and $\mathrm{CO}$ disks. The ${ }^{12} \mathrm{CO}$ emission is resolved while the $1.3 \mathrm{~mm}$ continuum appears only marginally resolved.
Table 1. Apparent sizes derived from PdBI data.

\begin{tabular}{ccc}
\hline \hline Observations & Flux & Gaussian $F W H M$ \\
\hline & $(\mathrm{mJy})$ & $\left({ }^{\prime \prime}\right)$ \\
$3.4 \mathrm{~mm}$ & $4.2 \pm 0.2$ & $0.6 \pm 0.2$ \\
$1.3 \mathrm{~mm}$ & $47.3 \pm 0.7$ & $0.42 \pm 0.02$ \\
\hline \multirow{2}{*}{$\mathrm{CO} J=2-1$} & $\left(\mathrm{Jy} \mathrm{km} \mathrm{s}{ }^{-1}\right)$ & $\left({ }^{\prime \prime}\right)$ \\
& $1.07 \pm 0.06$ & $0.87 \pm 0.06$ \\
\hline
\end{tabular}

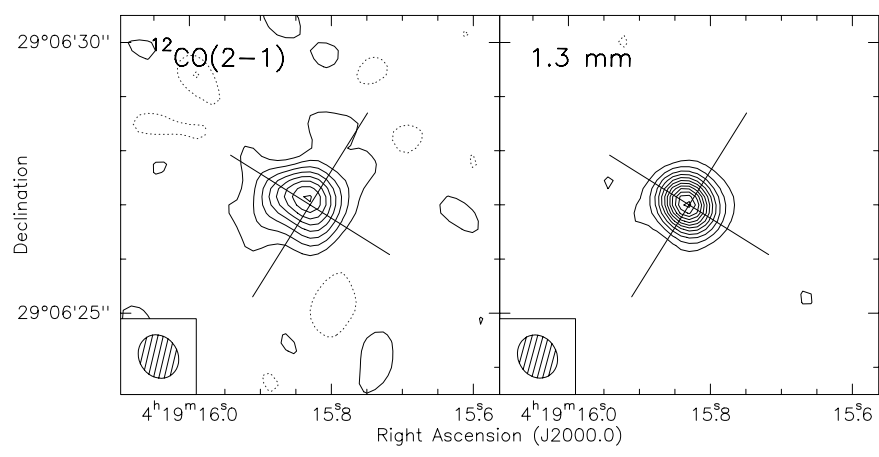

Fig. 2. Integrated ${ }^{12} \mathrm{CO} J=2 \rightarrow 1$ emission and $1.3 \mathrm{~mm}$ continuum emission towards BP Tau. Left: Integrated ${ }^{12} \mathrm{CO} J=2 \rightarrow 1$ emission; contour step is $60 \mathrm{mJy} \mathrm{km} \mathrm{s}{ }^{-1}$ /beam. Right: $1.3 \mathrm{~mm}$ continuum emission; contour step is $3 \mathrm{mJy} / \mathrm{beam}$.

With a value of $\alpha \simeq 2.7$, the spectral index of the continuum is fully consistent with thermal dust emission, mostly optically thin.

\subsection{0-m data}

In order to estimate the ${ }^{13} \mathrm{CO}$ column density inside the disk and to assess confusion from molecular clouds, we observed the ${ }^{12} \mathrm{CO} J=1 \rightarrow 0$ and $J=2 \rightarrow 1$ lines, and the ${ }^{13} \mathrm{CO} J=$ $2 \rightarrow 1$ line with the 30-m telescope in August 1999. Two lines were observed simultaneously using the dual frequency, dual polarization receivers of the $30-\mathrm{m}$ telescope. Total integration 

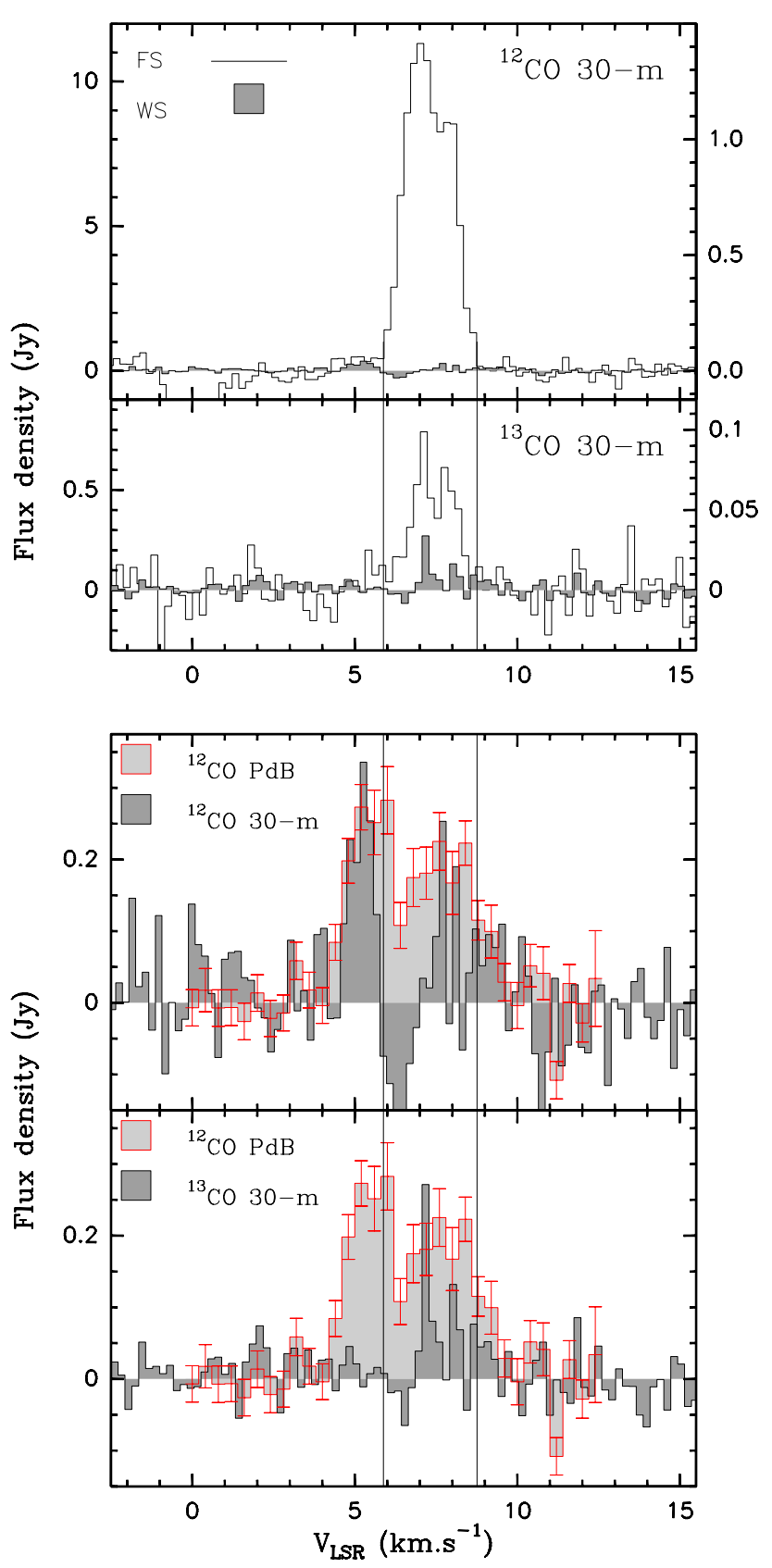

Fig. 3. Spectra of ${ }^{12} \mathrm{CO}$ and ${ }^{13} \mathrm{CO} J=2 \rightarrow 1$ towards BP Tau. From top to bottom: a) ${ }^{12} \mathrm{CO} J=2 \rightarrow 1$ observed in frequency switch (thin spectrum) and wobbler switch (shaded spectrum); b) ${ }^{13} \mathrm{CO} J=2 \rightarrow 1$ spectra in frequency switch (thin spectrum) and wobbler switch (shaded spectrum); c) superposition of the ${ }^{12} \mathrm{CO} J=2 \rightarrow 1$ integrated spectrum from the PdB observations (grey shaded histogram with error bars) and the wobbler switch ${ }^{12} \mathrm{CO} J=2 \rightarrow 1$ spectrum obtained with the 30-m (dark grey shaded histogram); d) superposition of the ${ }^{12} \mathrm{CO} J=2 \rightarrow 1$ integrated spectrum from the PdB observations (grey shaded histogram with error bars) and the wobbler switch ${ }^{13} \mathrm{CO} J=2 \rightarrow 1$ spectrum obtained with the $30-\mathrm{m}$ (dark grey shaded histogram). Comparison between PdBI and $30-\mathrm{m}$ data shows that emission from the disk is free of confusion outside the velocity range $[5.9-8.9] \mathrm{km} \mathrm{s}^{-1}$ (which is given by the thin vertical lines).

times were 45 hours for the ${ }^{12} \mathrm{CO} J=1 \rightarrow 0$ line, 33 hours for the ${ }^{13} \mathrm{CO} J=2 \rightarrow 1$ line and 13 hours for the ${ }^{12} \mathrm{CO} J=2 \rightarrow 1$ line. The last transition was observed to check the ability to detect the BP Tau disk despite the confusion with the molecular cloud along the line of sight.

We proceeded in two steps. We first took spectra in frequency switching mode to measure the total intensities of the lines. We then proceeded with long integrations in wobbler switching (with an offset of $30^{\prime \prime}$ ) which removes the signal of most of the surrounding material, because of the apparent rotation of the reference beam on the sky. Results are presented in Fig. 3 and in Table 2 . The $30-\mathrm{m}^{12} \mathrm{CO} J=2 \rightarrow 1$ spectrum suffers from confusion in the velocity range $6-9 \mathrm{~km} \mathrm{~s}^{-1}$, but the line wings agree very well with the spectrum obtained from the interferometric measurement (see Fig. 3). For the ${ }^{13} \mathrm{CO} J=2 \rightarrow 1$, where confusion is much more limited, there is no detectable signal in the beam switch spectrum of the 30-m, except for two weak narrow $\left(0.5 \mathrm{~km} \mathrm{~s}^{-1}\right)$ residual lines at the cloud velocities. In particular, in the blue wing of the BP Tau disk emission, where no confusion occurs, we only get a $3 \sigma$ limit in ${ }^{13} \mathrm{CO} J=2 \rightarrow 1$ of $0.12 \mathrm{Jy} \mathrm{km} \mathrm{s}^{-1}$.

\subsection{Comparison of the $P d B$ and $30-m$ observations}

Comparison of the 30-m frequency switch and wobbler switch spectra (Figs. 3a,b) clearly shows that BP Tau is embedded in or lying in front of a molecular cloud. The cloud actually has two velocity components, at 7.03 and $8.00 \mathrm{~km} \mathrm{~s}^{-1}$ respectively. The measured antenna temperatures of the ${ }^{12} \mathrm{CO} J=$ $1 \rightarrow 0(2.35 \mathrm{~K}$, not shown) and $J=2 \rightarrow 1(1.35 \mathrm{~K}$, see Fig. 3a) indicate a low density medium with sub-thermal excitation. The $\left[{ }^{12} \mathrm{CO}\right] /\left[{ }^{13} \mathrm{CO}\right]$ line intensity ratios are 23 and 14 for both components, which, considering possible enhancement of ${ }^{13} \mathrm{CO}$ abundance by fractionation effects, indicates essentially optically thin ${ }^{12} \mathrm{CO} J=2 \rightarrow 1$ emission $(\tau \leq 1-2)$. The ${ }^{13} \mathrm{CO} J=2 \rightarrow 1$ line appears completely optically thin.

From the LVG curves of Goldsmith et al. (1983), we derive an $\mathrm{H}_{2}$ density of $\sim 2 \times 10^{3} \mathrm{~cm}^{-3}$, and an $X[\mathrm{CO}] / \mathrm{d} V / \mathrm{d} r$ of order $10^{-6}$. Using the Taurus abundance for $\mathrm{CO}$ relative to $\mathrm{H}_{2}$, $7 \times 10^{-5}$ (Cernicharo \& Guélin 1987), and the line width of $0.7 \mathrm{~km} \mathrm{~s}^{-1}$, we find an $\mathrm{H}_{2}$ column density of $4 \times 10^{19} \mathrm{~cm}^{-2}$, corresponding to $A_{V} \simeq 0.05$. However, at such low extinctions, $\mathrm{CO}$ is much less abundant. Using $X[\mathrm{CO}]=5 \times 10^{-6}$ would give $A_{V} \simeq 0.5$, in agreement with the extinction measured by Hartmann et al. (1998). The BP Tau disk could thus be equally located behind one of the molecular clouds (or both), or in front of them if the extinction is intrinsic to the disk.

Given the small cloud opacity in the ${ }^{12} \mathrm{CO} J=2 \rightarrow 1$ line, emission from the BP Tau disk, whose systemic velocity is $6.70 \pm 0.05 \mathrm{~km} \mathrm{~s}^{-1}$, is free of any confusion at velocities outside the [5.9-8.9] $\mathrm{km} \mathrm{s}^{-1}$ range (see Fig. 3). Between 5.9 and $8.9 \mathrm{~km} \mathrm{~s}^{-1}$, the appearance of the BP Tau disk in ${ }^{12} \mathrm{CO}$ could be distorted by absorption by the molecular cloud. We have checked that the apparent symmetry of the ${ }^{12} \mathrm{CO} J=2 \rightarrow 1$ profile (see Fig. 3) and images (see Fig. 1) obtained with the interferometer implies that absorption from any of the two velocity component of the molecular cloud has an opacity less than 0.3 , and contaminates the emission at a level at most comparable to the noise. Parameters derived from the interferometer data are 
Table 2. 30-m data, line parameters.

\begin{tabular}{|c|c|c|c|c|}
\hline \multicolumn{5}{|c|}{$7.0 \mathrm{~km} \mathrm{~s}^{-1}$ cloud } \\
\hline${ }^{12} \mathrm{CO}$ & $\begin{array}{l}\text { Area } \\
\mathrm{K} \mathrm{km} \mathrm{s}^{-1}\end{array}$ & $\begin{array}{l}V_{\mathrm{LSR}} \\
\mathrm{km} \mathrm{s}^{-1}\end{array}$ & $\begin{array}{l}\text { Width } \\
\mathrm{km} \mathrm{s}^{-1}\end{array}$ & $\begin{array}{l}\text { Peak } \\
\text { K }\end{array}$ \\
\hline$J=1 \rightarrow 0$ & $3.26 \pm 0.02$ & $7.02 \pm 0.01$ & $1.25 \pm 0.01$ & 2.34 \\
\hline $\begin{array}{l}J=2 \rightarrow 1 \\
{ }^{13} \mathrm{CO}\end{array}$ & $1.80 \pm 0.05$ & $7.00 \pm 0.02$ & $1.15 \pm 0.03$ & 1.36 \\
\hline$J=2 \rightarrow 1$ & $0.077 \pm 0.009$ & [7.02] & $0.95 \pm 0.16$ & 0.077 \\
\hline \multicolumn{5}{|c|}{$8.0 \mathrm{~km} \mathrm{~s}^{-1}$ cloud } \\
\hline${ }^{12} \mathrm{CO}$ & $\begin{array}{l}\text { Area } \\
\mathrm{K} \mathrm{km} \mathrm{s}^{-1}\end{array}$ & $\begin{array}{l}V_{\mathrm{LSR}} \\
\mathrm{km} \mathrm{s}^{-1}\end{array}$ & $\begin{array}{l}\text { Width } \\
\mathrm{km} \mathrm{s}^{-1}\end{array}$ & $\begin{array}{l}\text { Peak } \\
\mathrm{K}\end{array}$ \\
\hline$J=1 \rightarrow 0$ & $1.02 \pm 0.02$ & $8.01 \pm 0.01$ & $0.57 \pm 0.01$ & 1.66 \\
\hline $\begin{array}{l}J=2 \rightarrow 1 \\
{ }^{13} \mathrm{CO}\end{array}$ & $0.63 \pm 0.05$ & $7.99 \pm 0.02$ & $0.65 \pm 0.04$ & 0.91 \\
\hline$J=2 \rightarrow 1$ & $0.045 \pm 0.006$ & {$[8.00]$} & $0.69 \pm 0.11$ & 0.069 \\
\hline \multicolumn{5}{|c|}{ Disk emission* } \\
\hline$J=2 \rightarrow 1$ & $\begin{array}{l}\mathrm{Jy} \mathrm{km} \mathrm{s}^{-1} \\
<0.12 \text { at } 3 \sigma\end{array}$ & - & $\begin{array}{l}\mathrm{km} \mathrm{s}^{-1} \\
{[3.5]}\end{array}$ & $\begin{array}{l}\mathrm{K} \\
-\end{array}$ \\
\hline
\end{tabular}

${ }^{*}$ The $3 \sigma$ upper limit on the integrated area in ${ }^{13} \mathrm{CO} J=2 \rightarrow 1$ for the disk is derived from the noise level using the ${ }^{12} \mathrm{CO} J=2 \rightarrow 1$ linewidth from the PdBI. Peak intensities are antenna temperatures $T_{A}^{*}$.

Table 3. Distance dependence of the parameters.

\begin{tabular}{llll}
\hline \hline Variable & Proportionality & \multicolumn{1}{c}{ Value } & Unit \\
\hline$D$ & $\propto D$ & 140 & $\mathrm{pc}$ \\
$T_{100}$ & $\propto D^{q}$ & $52 \pm 4$ & $\mathrm{~K}$ \\
$n_{100}$ & $\propto D^{s}$ & $3.0 \times 10^{7} \pm 0.4 \times 10^{7}$ & $\mathrm{~cm}^{-3}$ \\
$R_{\text {out }}$ & $\propto D$ & $122 \pm 10$ & $\mathrm{AU}$ \\
$V_{100}$ & $\propto D^{0.5}$ & $3.35 \pm 0.25$ & $\mathrm{~km} \mathrm{~s}^{-1}$ \\
$M_{*}$ & $\propto D$ & $1.32 \pm_{0.12}^{0.20}$ & $M_{\odot}$ \\
\hline
\end{tabular}

thus essentially unbiased if the BP Tau disk is located behind the molecular cloud.

\section{Analysis of the PdBI data}

Many of the parameters we derive from our analysis depend on the radial extent of the disk, and hence the actual distance to BP Tau. Table 3 provides the distance dependence of the measured disk parameters, and their value at the average distance of the Taurus cluster, 140 pc (Kenyon et al. 1994).

To estimate the properties of the BP Tau disk, we used a standard disk model (e.g. Pringle 1981) and the $\chi^{2}$ disk fitting procedures described in Guilloteau \& Dutrey (1998). The $\mathrm{CO}$ analysis is performed on the data containing the continuum.

To model both the $\mathrm{CO}$ and the continuum data, we proceed as follows. We performed a $\chi^{2}$ minimization on the $\mathrm{CO}$ data and in a second step, we use the kinetic temperature derived from the $\mathrm{CO}$ analysis to model the continuum data at 3.4 and $1.3 \mathrm{~mm}$ (both the $\mathrm{CO}$ and the dust emissions are mostly optically thin and should trace the same region). In this way we obtain an estimate of the $\mathrm{H}_{2}$ mass and the density. This value is also used to determine the depletion factor $f(\mathrm{CO})=$ $X\left[{ }^{12} \mathrm{CO}\right] / X_{\mathrm{TMC} 1}\left[{ }^{12} \mathrm{CO}\right]$. We then iterate the minimization with these new parameters for the $\mathrm{CO}$ data and the continuum. The results are given in Table 4.

The $\chi^{2}$ fitting procedure indicates that the radial dependence of the velocity is $r^{-(0.52 \pm 0.04)}$, so the rotation is Keplerian. The disk is found to be small, with an outer radius of $120 \mathrm{AU}$. Although ${ }^{12} \mathrm{CO}$ usually allows direct determination of the disk kinematics, and hence of the stellar mass (Guilloteau \& Dutrey 1998; Simon et al. 2000), the analysis of the BP Tau disk is complicated by a combination of two facts.

First, the ${ }^{12} \mathrm{CO} J=2 \rightarrow 1$ line appears only moderately optically thick. For the best fit model, the peak optical depth of the ${ }^{12} \mathrm{CO} J=2 \rightarrow 1$ line is only 3 , and in most of the disk (i.e. at radii greater than about $50 \mathrm{AU}$ ) the line remains optically thin. Since in the optically thin regime and high temperature limit, the line emission is proportional at first order to $\Sigma(r) \times X(r) / T_{k}(r)$ (in the Rayleigh Jeans approximation, with constant linewidth and $X(r)$ being the molecular abundance with respect to $\mathrm{H}_{2}$, see Dartois et al. 2003), the limited optical depth of ${ }^{12} \mathrm{CO} J=2 \rightarrow 1$ line leads to a relatively strong coupling between the kinetic temperature distribution $\left(T_{k}(r), q\right)$ and the $\mathrm{CO}$ molecular density $(n(r) \times X(r), s)$.

Second, the disk is small and only moderately resolved. In such a case, there is a significant coupling between the brightness temperature distribution and the outer radius $R_{\text {out }}$. Steeper distributions can be compensated for by a larger radius; $\mathrm{CO}$ being optically thin, the outer brightness distribution goes with $p-q$, so that a steeper distribution means a lower value of $q$. A larger radius will require a higher disk inclination to maintain the same total line flux, and thus modify the derived stellar mass through the derivation of the $v \sin (i), i$ parameters. Unfortunately, this degeneracy is quite significant. Depending on the assumption on $q$, we found through minimizations that the stellar mass could vary between 1.3 and $0.6 M_{\odot}$ (for a distance $D=140 \mathrm{pc}$ ) when $q$ varied from 0.5 to -0.1 , corresponding to inclinations varying between $28^{\circ}$ and $43^{\circ}$ respectively. Assuming that the disk mid-plane and the star rotation axis are perpendicular, the stellar period (Bouvier et al. 1986) and the $V \sin i$ of the star (Hartman et al. 1986) can give an upper limit for the inclination angle, $i \leq 52^{\circ}$. This limit is consistent with our data, but does not allow us to select between the two extreme solutions.

This degeneracy is a significant limitation of the current data. To avoid consequences of this problem for the other disk parameters, we proceeded as follows. We fixed $q=0.3$, which was at all times the most positive value to be within $1 \sigma$ of the best fit in the $\left(T_{100}, q\right) \chi^{2}$ plane.

Once $q$ is fixed, the other couplings introduced by the partial optical thickness of $\mathrm{CO}$ are illustrated in Fig. 4, which displays $\chi^{2}$ surfaces showing the coupling between the $\mathrm{CO}$ depletion $f(\mathrm{CO})$ and $T_{k}$, and between $T_{k}$ and $R_{\text {out }}$. Since both couplings are relatively weak, $R_{\text {out }}$ is well determined in all cases.

Starting from this value of $R_{\text {out }}$, we proceeded to perform 2-parameter minimizations, with the density law exponent $s$ fixed: $\left(T_{100}, f(\mathrm{CO})\right),(V \sin (i), i),\left(\mathrm{d} V, R_{\text {out }}\right)$, and finally $\left(T_{100}, R_{\text {out }}\right)$. The whole process was iterated several times 
Table 4. Best parameters for the BP Tau disk (CO $J=2 \rightarrow 1$ data and continuum).

\begin{tabular}{|c|c|c|}
\hline Assumed distance & $D(\mathrm{pc})=140$ & \\
\hline Systemic velocity & $V_{\text {LSR }}\left(\mathrm{km} \cdot \mathrm{s}^{-1}\right)=6.71$ & \pm 0.05 \\
\hline Orientation & $\mathrm{PA}=57$ & $\pm 4^{\circ}$ \\
\hline Inclination & $i=28$ & $\pm 2^{\circ}$ \\
\hline Outer radius & $R_{\text {out }}(\mathrm{AU})=122$ & \pm 10 \\
\hline Turbulent linewidth & $\Delta v\left(\mathrm{~km} \mathrm{~s}^{-1}\right)=0.3$ & \pm 0.1 \\
\hline \multicolumn{3}{|c|}{ Abundance $\& \mathrm{H}_{2}$ density law: $n(r)=n_{100}\left(\frac{r}{100 \mathrm{AU}}\right)^{-s}$} \\
\hline${ }^{12} \mathrm{CO}$ reference ${ }^{(a)}$ & $X_{\mathrm{TMC} 1}^{12}=7 \times 10^{-5}$ & - \\
\hline${ }^{12} \mathrm{CO}$ abundance & $X\left({ }^{12} \mathrm{CO}\right)=4.3 \times 10^{-7}$ & $\pm 0.4 \times 10^{-7}$ \\
\hline${ }^{12} \mathrm{CO}$ depletion & $f\left({ }^{12} \mathrm{CO}\right)=160$ & \pm 20 \\
\hline Density $^{(b)}$ & & \\
\hline at $100 \mathrm{AU}$ & $n_{100}\left(\mathrm{~cm}^{-3}\right)=3.0 \times 10^{7}$ & $\pm 0.4 \times 10^{7}$ \\
\hline exponent & $s=3.0$ & \pm 0.2 \\
\hline \multicolumn{3}{|c|}{ Temperature law: $\quad T(r)=T_{100}\left(\frac{r}{100 \mathrm{AU}}\right)^{-q}$} \\
\hline Temperature $^{(c)}$ & & \\
\hline at $100 \mathrm{AU}$ & $T_{100}(\mathrm{~K})=52$ & \pm 4 \\
\hline exponent & $q \simeq 0.0-0.5$ & \\
\hline assumed value & $q=0.3$ & \\
\hline \multicolumn{3}{|c|}{ Velocity law: $\quad V(r)=V_{100}\left(\frac{r}{100 \mathrm{AU}}\right)^{-v}$} \\
\hline Velocity at $100 \mathrm{AU}$ & $V_{100}\left(\mathrm{~km} \mathrm{~s}^{-1}\right)=3.35$ & \pm 0.25 \\
\hline Velocity exponent & $v=0.52$ & \pm 0.04 \\
\hline Stellar mass & $M_{*}\left(M_{\odot}\right)=1.32$ & $\pm_{0.12}^{0.20}$ \\
\hline
\end{tabular}

\begin{tabular}{|c|c|c|}
\hline \multicolumn{3}{|c|}{ Surface density law: $\Sigma(r)=\Sigma_{100}\left(\frac{r}{100 \mathrm{AU}}\right)^{-p}$} \\
\hline Surface Density ${ }^{(d)}$ & & \\
\hline at $100 \mathrm{AU}$ & $\Sigma_{100}\left(\mathrm{~cm}^{-2}\right)=1.3 \times 10^{22}$ & $0.2 \times 10^{22}$ \\
\hline & $\Sigma_{100}\left(\mathrm{~g} \mathrm{~cm}^{-2}\right)=0.06$ & 0.01 \\
\hline exponent & $p \simeq 1.7$ & $\simeq 0.2$ \\
\hline
\end{tabular}

\begin{tabular}{|c|c|c|}
\hline \multicolumn{3}{|c|}{ Scale height law: $\quad H(r)=H_{100}\left(\frac{r}{100 \mathrm{AU}}\right)^{-h}$} \\
\hline Scale height & & \\
\hline at $100 \mathrm{AU}$ & $H_{100}(\mathrm{AU})=17$ & - \\
\hline exponent & $h \simeq 1.35$ & - \\
\hline
\end{tabular}

\begin{tabular}{|c|c|c|}
\hline & Dust: $\kappa_{v}=\kappa_{o} \times\left(\frac{v}{10^{12} \mathrm{~Hz}}\right)^{\beta}$ & \\
\hline Absorption law & $\kappa_{o}=0.1$ & \\
\hline Dust exponent & $\beta=0.70$ & \pm 0.05 \\
\hline Dust disk size & $R_{\mathrm{d}}(\mathrm{AU})>100$ & \\
\hline total mass & $M_{\mathrm{d}}\left(M_{\odot}\right) \sim 1.2 \times 10^{-3}$ & \\
\hline
\end{tabular}

The errors are the $1 \sigma$ formal errors from the $\chi^{2}$ fit.

(a) $X_{\mathrm{TMCl}}$, the ${ }^{12} \mathrm{CO}$ abundance in TMC 1 is taken from Cernicharo \& Guélin 1987. ${ }^{(b, c, d)}$ The temperature is given for $q=0.3$. The error on the density and temperature do not take into account the coupling with the temperature exponent $q$.

The $\mathrm{CO}$ abundance in the disk $X\left({ }^{12} \mathrm{CO}\right)$ or the depletion $f\left({ }^{12} \mathrm{CO}\right)=$ $X\left({ }^{12} \mathrm{CO}\right) / X_{\mathrm{TMC} 1}^{12}$ is obtained by reference to the total disk mass measured from the continuum data (dust).

to guarantee convergence. At each step, $\left(T_{100}, q\right)$ was checked to verify that $q=0.3$ was within $1 \sigma$.
The above process does not allow a separation of $X(r)$ (or $f(\mathrm{CO})$ ) from $\Sigma(r)$. We use the continuum emission from dust to provide an independent measurement of $\Sigma(r)$. Dust emission being optically thin at such wavelengths, the continuum is proportional to $\kappa_{v} \times \Sigma(r) \times T_{k}(r)$. Using $\kappa_{v}(v)=0.1\left(v / 10^{12} \mathrm{~Hz}\right)^{\beta}$ (see Beckwith et al. 1990), the measurements at $3.4 \mathrm{~mm}$ and $1.3 \mathrm{~mm}$ allow us to constrain $\beta$, and thus $\kappa_{v}$. Since $T_{k}(r)$ has been measured from the $\mathrm{CO}$ emission, $\Sigma(r)$ is determined. The dust emission also directly constrains $p+q$ (see Pietu et al. 2003). We find $p+q=1.8 \pm 0.1$. Since the density exponent is $s=p+1.5-q / 2$, this value is in agreement with (but more accurate than) that derived from the measurement of $s$ and $q$ from ${ }^{12} \mathrm{CO}$, which corresponds to $p+q=s-1.5+3 q / 2 \simeq 2.2 \pm 0.4$.

Since $q$ is rather poorly constrained, $p$ ranges from $p \simeq 1.2$ (for $q \simeq 0.6$ as in DM Tau or GM Aur Guilloteau \& Dutrey 1998; Dutrey et al. 1998), to $p \simeq 1.5$ for our assumed value of $q=0.3$. The total mass derived from the nominal best model is $1.2 \times 10^{-3} M_{\odot}$, and the surface density at $100 \mathrm{AU} 0.06 \mathrm{~g} \mathrm{~cm}^{-2}$ (of dust and gas).

\section{Discussion}

In this section, we first present the new stellar mass estimate and then we discuss the physical parameters of this disk, with respect to the limitation of the data, and compare them to the properties of disk encountered around other single CTTs such as DM Tau (Guilloteau \& Dutrey 1998) or GM Aur (Dutrey et al. 1998).

\subsection{The CO dynamical mass estimate}

The estimate for the mass of BP Tau that we determine, $M_{*}=1.32 \pm_{0.12}^{0.20} \quad M_{\odot}$ (Table 3 ), at the 140 pc fiducial distance, is consistent with our earlier estimate, $1.24 \pm_{0.25}^{0.32} M_{\odot}$ (SDG). However, these error bars do not include the coupling with other parameters. The coupling between $q$ and $R_{\text {out }}$ will result in lower values for $M_{*}$ for smaller $q$, and higher values for larger $q$. For $q=-0.1$, the derived mass becomes $M_{*}=0.60 \pm 0.06 M_{\odot}$.

Figure 5 shows these two extreme values of BP Tau's distance-independent parameter $L / M_{*}^{2}$ plotted on a modified $\mathrm{H}$ $\mathrm{R}$ diagram in the same format as in Fig. 3 of SDG. We use estimates of BP Tau's luminosity, $0.93 L_{\odot}$, and spectral type, K7, from Gullbring et al. (1998), as before. The $L / M^{2}$ value lies between D'Antona \& Mazzitelli's (1997) tracks for 0.5 and $0.6 M_{\odot}$ stars, on the $0.8 M_{\odot}$ track of Baraffe et al. (1998), and similarly for the tracks calculated by Palla \& Stahler (1999) and Siess et al. (2000). Our mass uncertainty rather affects the distance and the age determination, since the dynamical mass obtained by our technique scales linearly with distance (Table 3). Using $M_{*}=0.8$ from the evolutionary tracks, the distance derived from the dynamical mass is $90 \pm 20 \mathrm{pc}$ for $q=0.3$, but becomes larger for smaller values of $q$. This is a hint of, but not a strong argument for, a distance somewhat closer than 140 pc (e.g. Favata et al. 1998, and see also Bertout et al. 1999). 
(a)

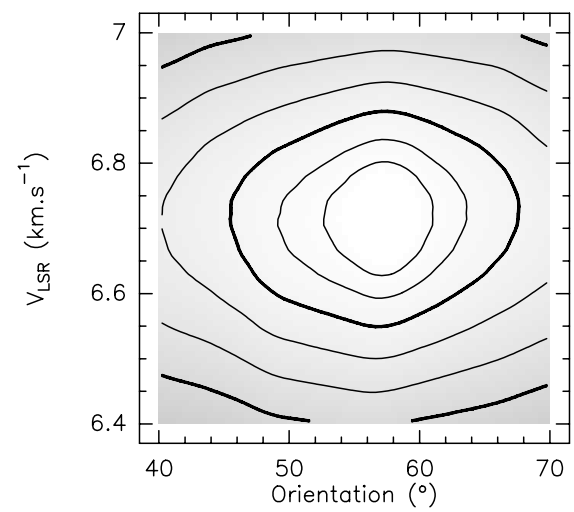

(d)

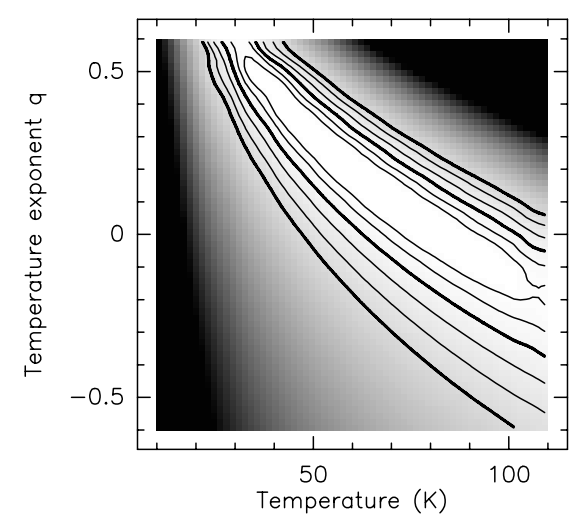

(g)

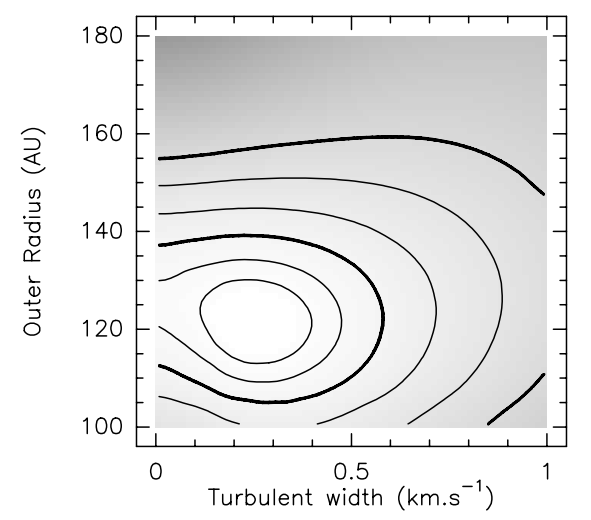

(b)
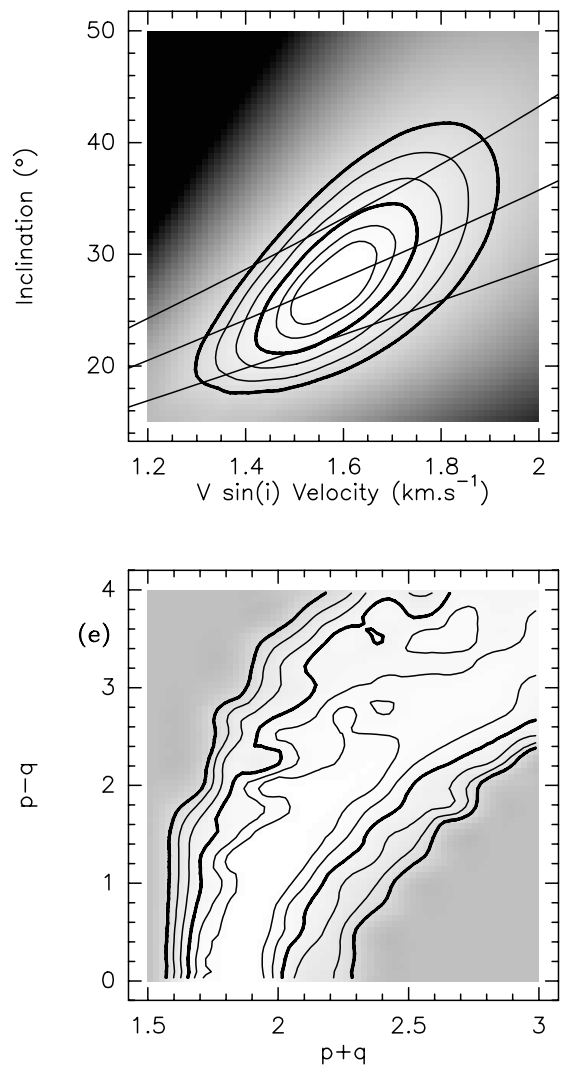

(h)

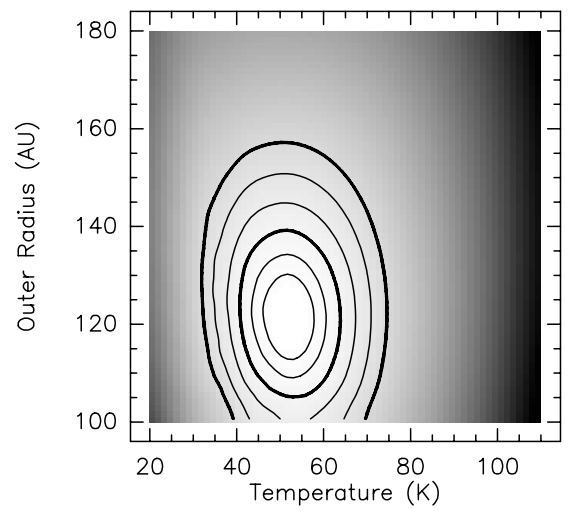

(e)

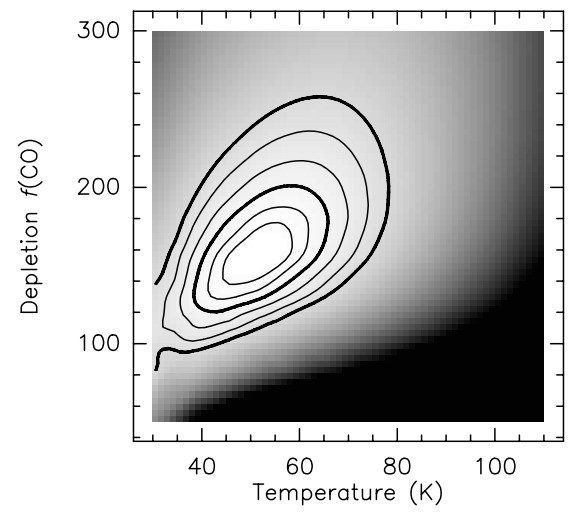

(f)

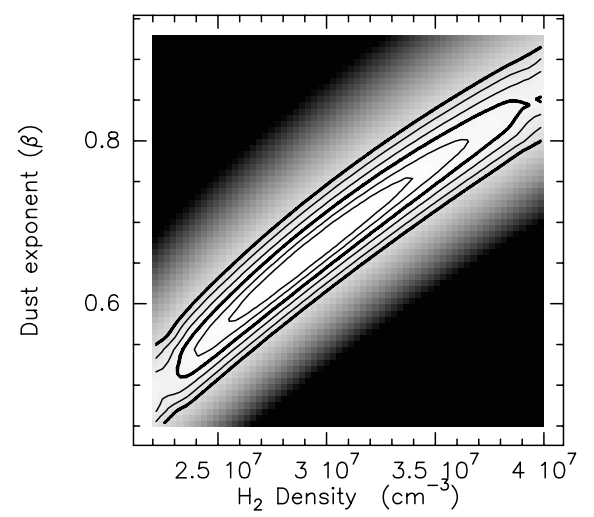

(i)

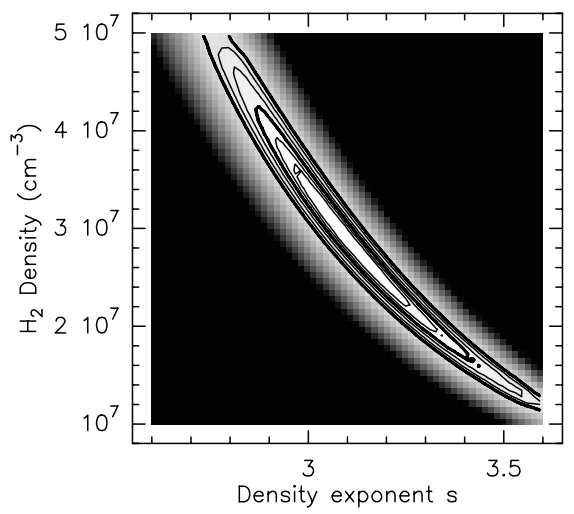

Fig. 4. $\chi^{2}$ surfaces for pairs of parameters. Contours are spaced by $1 \sigma$, with the $3 \sigma$ and $6 \sigma$ contours in thick lines. The 3 curves on panel b) correspond to stellar masses of $0.96,1.32$ and $1.92 M_{\odot}$, corresponding to $\pm 3 \sigma$ on the stellar mass determination.

It is worth stressing that all the other sources in the SDG sample possess optically thick $\mathrm{CO}$ disks that are much larger and brighter, so their outer radius, temperature law $(q)$, inclination and hence stellar masses are quite reliably determined.

\subsection{Disk properties}

The BP Tau disk differs from all the other disks imaged in ${ }^{12} \mathrm{CO}$ so far in two main aspects: 1) both $\mathrm{CO}$ and dust emissions are very compact, and 2) the data itself, as we argue in Sect. 2.3, shows that the $\mathrm{CO}$ is optically thin. Because of the latter point, the radial profiles of density and temperature are coupled and the analysis is complicated by the small angular size of the disk.

\subsubsection{Optically thin $\mathrm{CO}$ emission}

The low optical depth may be demonstrated by either 1) comparing the disk mass derived from the upper limit in ${ }^{13} \mathrm{CO} J=$ $2 \rightarrow 1$ from the $30-\mathrm{m}$ telescope and the flux density at $1.3 \mathrm{~mm}$ or 2 ) using the physical disk model derived from the ${ }^{12} \mathrm{CO}$ interferometric data.

For the first calculation, we take the flux densities given in Tables 2 and 4 . We assume the ${ }^{13} \mathrm{CO}$ abundance found in TMC1 $\left(X_{\mathrm{TMC} 1}^{13}=10^{-6}\right)$ and a standard dust absorption coefficient with a gas-to-dust ratio of 100 (Beckwith et al. 1990, see also Table 3 ). The kinetic temperature is derived from the CO model: we use $T_{\text {rot }}=60 \mathrm{~K}$ as a mean value throughout the disk. A lower limit on the depletion factor $f(\mathrm{CO})$ is to a first 


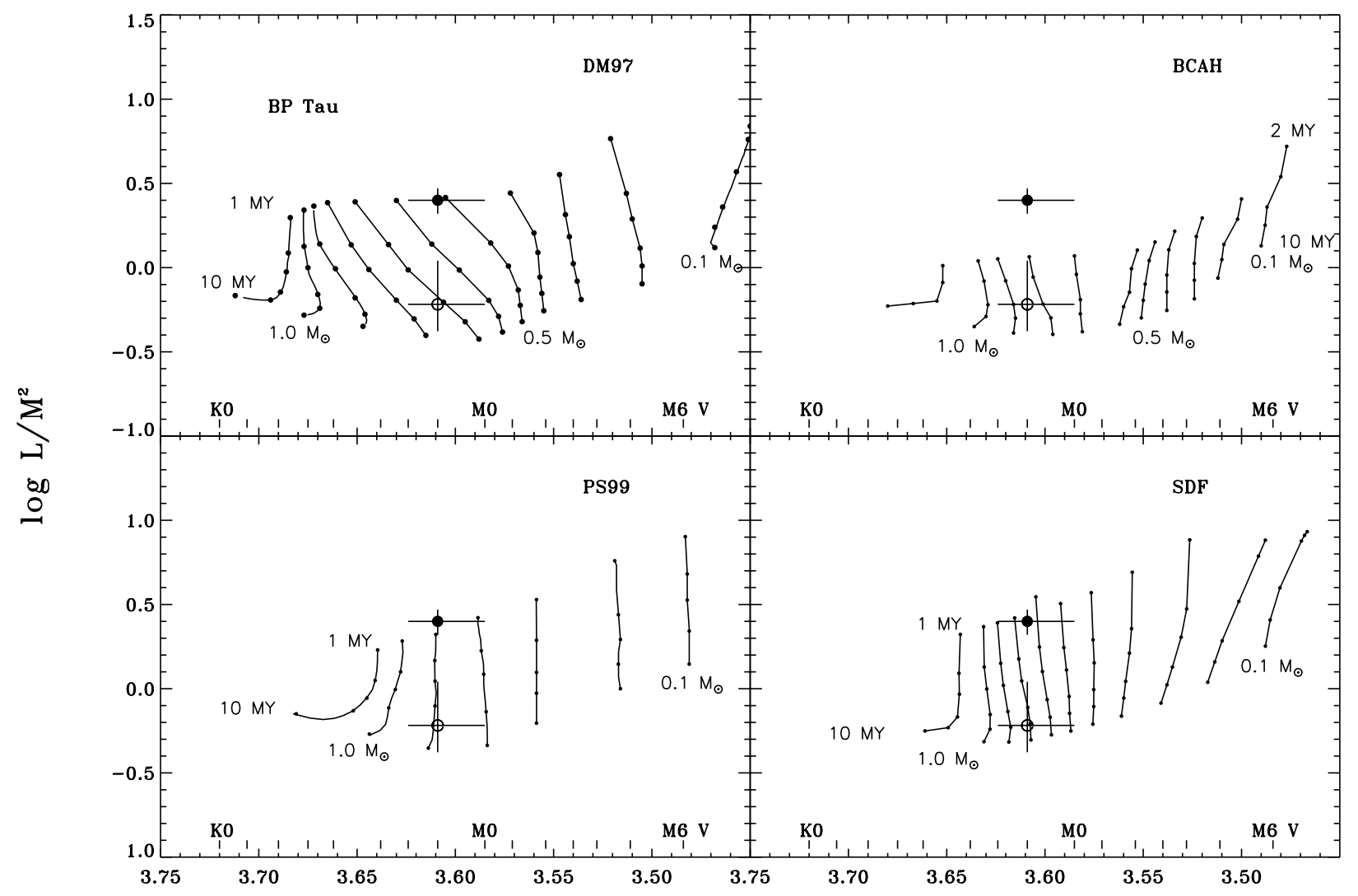

\section{$\log \mathrm{T}_{\text {eff }}$}

Fig. 5. The location of BP Tau in the $\left(L / M^{2}\right.$ vs. $\left.T_{\text {eff }}\right)$ diagram using the two extreme dynamical mass determinations in this work (for $q=-0.1$ (filled circle) and $q=0.3$ (open circle)). The theoretical evolutionary tracks are presented in the same way as in Fig. 3 of SDG. Upper left-hand panel: Tracks calculated by d'Antona \& Mazzitelli (1997) for stars of mass $0.1,0.2,0.3,0.4,0.5,0.6,0.7,0.8,0.9,1.0$, and $1.2 M_{\odot}$; the dots indicate ages 1, 2, 3, 5, 7, and 10 Myr. Upper right-hand panel: Same but for tracks calculated by Baraffe et al. (1998); the dots indicate ages 2, 3, 5, 7, and 10 Myr. Lower left: Same but for calculations by Palla \& Stahler (1999) for stars of mass 0.1, 0.2, 0.4, 0.6, 0.8, 1.0, and 1.2 $M_{\odot}$ at indicated ages 1, 2, 3, 5, and 10 Myr. Lower right: Same for calculations by Siess et al. (2000) for the same masses and ages as in the upper left.

order given by the ratio of the $\mathrm{H}_{2}$ mass estimates derived from the thermal dust emission and the ${ }^{13} \mathrm{CO} J=2 \rightarrow 1$ line.

The mass derived from ${ }^{13} \mathrm{CO} J=2 \rightarrow 1, M_{13}\left(\mathrm{H}_{2}\right)$ is given by:

$M_{13}\left(\mathrm{H}_{2}\right)=\frac{\int S_{13} \cdot \mathrm{d} v}{2 k T_{\text {rot }}}\left(\frac{c}{v}\right)^{2} \times \frac{D^{2}}{\kappa_{v}} M_{\odot}$

with $\kappa_{v}$ the absorption coefficient for the transition $J+1 \rightarrow J$ :

$$
\begin{aligned}
\kappa_{v}= & \frac{8 \pi^{3} \mu^{2}}{3 h \mathrm{~d} v} \frac{\left(\mathrm{e}^{\frac{-h v_{0} J(J+1)}{2 k T_{\mathrm{rot}}}}-\mathrm{e}^{\frac{-h \nu_{\mathrm{o}}(J+1)(J+2)}{2 k T_{\mathrm{rot}}}}\right)}{Z} \\
& \times \frac{(J+1)}{m_{\mathrm{w}} m\left(\mathrm{H}_{2}\right)} X_{\mathrm{TMC} 1}\left({ }^{13} \mathrm{CO}\right) .
\end{aligned}
$$

Where $v_{\mathrm{o}}=v_{1 \oplus}, d v=1 \mathrm{~km} \mathrm{~s}^{-1}, m_{\mathrm{W}}$ is the mean molecular weight $\left(m_{\mathrm{w}}=1.3\right)$ and $Z$ the partition function $(Z=23$ for $\left.T_{\text {rot }}=60 \mathrm{~K}\right)$. For the ${ }^{13} \mathrm{CO} J=2 \rightarrow 1$ transition we get $\kappa_{v}=$ $4.7 \mathrm{~cm}^{2} \mathrm{~g}^{-1}$ and the total disk mass is equal to $M_{13}\left(\mathrm{H}_{2}\right)=2.7 \times$ $10^{-5} M_{\odot}$. This leads to a lower limit on $f\left({ }^{13} \mathrm{CO}\right) \sim 50$.
Alternatively, we can also obtain this result using the physical disk model derived from the ${ }^{12} \mathrm{CO}$ interferometric data. The $30-\mathrm{m}^{13} \mathrm{CO}$ line flux from the disk is less than $0.12 \mathrm{Jy} \mathrm{km} \mathrm{s}^{-1}$ (at $3 \sigma$ ). The best disk model derived from the ${ }^{12} \mathrm{CO}$ data predicts a flux of $0.025 \mathrm{Jy} \mathrm{km} \mathrm{s}^{-1}$ in ${ }^{13} \mathrm{CO} J=2 \rightarrow 1$. Hence, the ${ }^{13} \mathrm{CO} J=2 \rightarrow 1$ data constrains the $\mathrm{CO}$ depletion $f\left({ }^{13} \mathrm{CO}\right)$ to be higher than $f\left({ }^{12} \mathrm{CO}\right) /(0.12 / 0.025)=40\left(f\left({ }^{12} \mathrm{CO}\right)\right.$ is given in Table 4).

Using the same method for DM Tau, $f(\mathrm{CO})$ is found around 10 by Dartois et al. (2003). Moreover, contrary to BP Tau, the ${ }^{12} \mathrm{CO} J=2 \rightarrow 1$ emission in the DM Tau disk is strongly optically thick.

\subsubsection{Density distribution}

In the modelling, the total disk mass, the $\mathrm{CO}$ depletion factor and therefore $n_{100}$ the density at $100 \mathrm{AU}$ are derived from the fit of the continuum data (Sect. 3 and Table 4). First of all, the continuum fit at 3.4 and $1.3 \mathrm{~mm}$ reveals that the dust spectral index $\beta$ has a value of $0.70 \pm 0.05$, somewhat smaller than the 
average found for other T Tauri disks $(\beta=0.95 \pm 0.11$, Dutrey et al. 1996). The disk mass is relatively low $\left(\sim 1.2 \times 10^{-3} M_{\odot}\right)$. This low value is a result of a lower density, rather than of the small size of the disk. Surface density laws have been estimated in some disks from the optically thin dust emission by Dutrey et al. (1996), whose results suggest that the dust distribution follows shallow surface density laws with $p \simeq 1-1.5$ and typical values for $\Sigma_{100}$ around $5 \times 10^{23} \mathrm{~cm}^{-2}$. Compared to these disks (most of them also possess large CO disks), $\Sigma_{100}$ in BP Tau is significantly lower, by a factor 40 , but the surface density exponent is similar $(p \simeq 1.2-1.5)$.

\subsubsection{Temperature distribution}

Taking $q=0.3$ as the most probable value, the temperature at $100 \mathrm{AU}$ is $T_{100}=52 \mathrm{~K}$. However, the rather large uncertainty on $q$ allows $T_{100}$ to vary from 35 (for $q=0.5$ ) to $>100 \mathrm{~K}$ (for $q<0$ ) (see Fig. 4). It is possible in special cases to have $q<0$ around the disk mid-plane (e.g. Fig. 3 from D'Alessio et al. 1999). However, since the CO $J=2-1$ emission is optically thin, it samples the whole disk, and measures a (vertically) averaged temperature gradient, for which $q$ is unlikely to be negative. Marginally lower temperatures can be obtained by allowing the depletion $f(\mathrm{CO})$ to be $\leq 150$ (see Fig. 4). This is somewhat hot for a K7 star: the expected blackbody temperature at $100 \mathrm{AU}$ would be only 26-38 K, depending on the assumed disk flaring. The same analysis performed on the CO disks of the K 7 star GM Aur (Dutrey et al. 1998) or the M 1 star DM Tau (Guilloteau \& Dutrey 1998) provide $T_{k}(r) \simeq 30 \times(r / 100 \mathrm{AU})^{-0.65} \mathrm{~K}$, as expected for flared passive disks heated by the central stars. In typical disks, because of the opacity of the CO line, $T_{100}$ is representative of a region located at about 2-4 scale heights above the disk mid-plane (Dartois et al. 2003), and the temperature in the disk plane is lower ( $\simeq 13 \mathrm{~K}$ from Dartois et al. 2003, see also d'Alessio et al. 1999). In BP Tau where the CO emission is partly optically thin, $T_{100}$ is closer to an average value throughout the disk vertical structure. The BP Tau disk thus appears hotter than the others.

Neither the viscous heating, which may only be efficient within the 10-20 central AU, nor the differences in stellar luminosities between BP Tau $\left(0.93 L_{\odot}\right)$ and GM Aur $\left(0.74 L_{\odot}\right.$, SDG) are enough to explain such a value for $T_{100}$. It is more likely due to a combination of several effects linked to the low angular resolution which complicates the $\mathrm{CO}$ analysis and does not allow us to provide accurate estimates of $q$ (see Sect. 3). However, it also might be partly related to the lower disk mass of BP Tau, and to active grain growth. If the dust opacity in the visible and near IR is much smaller in BP Tau than either in GM Aur and DM Tau, because of the lower disk mass and perhaps also because of different grain properties, it is possible that the "superheated" layer (Chiang \& Goldreich 1997) occupies a significant fraction of the disk for BP Tau. In the extreme limit where $\tau_{V} \simeq 1$, and with $\tau_{\mathrm{IR}} \ll \tau_{V}$, the whole disk could be heated above the blackbody temperature.

To do so, only a tiny fraction of small grains should still be in the disk. Small grains of $a \simeq 0.1 \mu \mathrm{m}$ have
$\kappa_{V} \approx 4 \times 10^{4} \mathrm{~cm}^{2} \mathrm{~g}^{-1}$ (for dust only, Chiang \& Goldreich 1997). Assuming $\Sigma_{\text {dust }}(r)=\Sigma_{0 d}(r / 100 \mathrm{AU})^{-1.5}$, an opacity $\tau_{V}(100 \mathrm{AU})=1$ at the disk plane is reached for $\Sigma_{0 d}=$ $5 \times 10^{-5} \mathrm{~g} \mathrm{~cm}^{-2}$ (of small grains), which corresponds to a mass of small grains around $8 \times 10^{-7} M_{\odot}$. Since the total dust mass derived from mm continuum emission is $\simeq 1.2 \times 10^{-5} M_{\odot}$, the fraction of small grains should not exceed $6 \times 10^{-2}$.

Unfortunately, imaging such an optically thin disk requires a very high sensitivity and is probably out of the possibilities of current adaptive optics systems on large telescopes. Detailed modelling of the BP Tau SED in the Visible and in the NIR may confirm or not our hypothesis.

\subsection{A clearing disk?}

Our analysis shows that the CO disk of BP Tau differs from the large $\mathrm{CO}$ and dust disks previously studied by mm interferometry. This is somewhat surprising because BP Tau presents in the optical and infrared all the characteristics of a CTTs, including a relatively strong accretion rate. At mm wavelengths, the BP Tau disk can be considered as atypical for the main following reasons:

1. its $\mathrm{CO}$ and dust disk is small and faint;

2. the ${ }^{12} \mathrm{CO} J=2 \rightarrow 1$ transition is optically thin;

3. with respect to the dust, $\mathrm{CO}$ is depleted by a large factor.

Points 1 and 2 are important with respect to the sensitivity of current $\mathrm{mm}$ arrays. The detection of the CO disk of BP Tau took about 25 hours of integration time compared to 4 hours for a "standard" large CO disk. Interferometers now in operation (and a fortiori large single-dish telescopes) may fail to detect most of such disks.

Concerning point 3 , we assume a gas-to-dust ratio of 100 , as usually made in these kinds of studies. With a lower gas-todust ratio, the $\mathrm{CO}$ depletion should be accordingly less important with respect to $\mathrm{H}_{2} . \mathrm{H}_{2}$ itself could be depleted with respect to the dust but we cannot determine this without a direct detection of $\mathrm{H}_{2}$. The dust absorption coefficient remains also another possible source of uncertainty. Nevertheless, the CO depletion factor we have derived is significantly higher than in other TTauri disks. The low $\mathrm{CO}$ abundance may actually be a consequence of higher photodissociation resulting from the low $\mathrm{H}_{2}$ surface density. It cannot be due to condensation of $\mathrm{CO}$ on dust grains, since the mean temperature is higher than $50 \mathrm{~K}$, well above the $\mathrm{CO}$ freezing temperature $(17 \mathrm{~K})$.

All these points argue in favor of a disk which has started to clear a significant fraction of its primary gas and dust. This statement is reinforced by the relatively high temperature of the disk, which might be explained by lower opacities of the dust in the visible and in the near infrared. It is interesting to note that the only other known disk with similar outer disk properties (small outer radius and small mm flux) has been discovered around V 836 Tau, an object presenting all the characteristics of being in the stage of inner disk dissipation from the optical data (Duvert et al. 2000). The BP Tau disk is however different because it still has a very active inner disk. This suggests that inner and outer disks may not begin to dissipate simultaneously. Finally, the CO content of BP Tau cannot result from 
evaporation of proto-comets. Large active comets such as HaleBopp contain about $10^{36}$ evaporated $\mathrm{CO}$ molecules (Biver et al. 1999). Since the BP Tau disk mass is $1.2 \times 10^{-3} M_{\odot}$ and the $\mathrm{CO}$ abundance $3.7 \times 10^{-7}$, the disk contains about $2 \times 10^{47}$ CO molecules. Thus a few times $10^{11}$ large comets like Hale Bopp would be required. Therefore the $\mathrm{CO}$ gas must be the remnant of the primordial nebula.

\section{Summary}

Using the standard method we used to study Class II protoplanetary disks, we have analyzed the ${ }^{12} \mathrm{CO} J=2 \rightarrow 1$ and continuum observations at 3.4 and $1.3 \mathrm{~mm}$ of BP Tau. We find that:

- BP Tau is surrounded by a compact $\left(R_{\text {out }} \simeq 120 \mathrm{AU}\right)$ dust and gas disk.

- The disk exhibits direct evidence for Keplerian rotation, around a star of mass $M_{*}=(1.3 \pm 0.2)(D / 140 \mathrm{pc}) M_{\odot}$.

- The ${ }^{12} \mathrm{CO} J=2 \rightarrow 1$ line appears only marginally optically thick.

- The temperature is relatively high, about $50 \mathrm{~K}$ at $100 \mathrm{AU}$.

- The mm continuum emission is consistent with the thermal emission from circumstellar dust with an emissivity index $\beta \simeq 0.7$, with a disk mass about $1.2 \times 10^{-3} M_{\odot}$.

- Assuming a gas-to-dust ratio of 100, the CO appears to be depleted by a factor of order 150 with respect to $\mathrm{H}_{2}$.

The unusual properties of the circumstellar disk suggest that BP Tau may be a transient object, in the process of clearing its disk. This object might belong to a class of intermediate disks between Class II and Class III that we fail to detect today due to the lack of sensitivity of mm arrays.

Only ALMA has the potential to enable systematic surveys of such objects around stars having a continuum flux at $1.3 \mathrm{~mm}$ of order $S_{v} \sim 30-50 \mathrm{mJy}$ or less.

Acknowledgements. We acknowledge all the Plateau de Bure IRAM staff for their help during the observations. We thank Claude Bertout for useful discussion on the distance measurement with Hipparcos, and Jacques Crovisier for discussion on the $\mathrm{CO}$ in comets. The work of M.S. was supported in part by NSF Grant 98-19694.

\section{References}

D’Alessio, P., Calvet, N., Hartmann, L., Lizano, S., \& Cantó, J. 1999, ApJ, 527, 893 (dA99)
Baraffe, I., Chabrier, G., Allard, F., \& Hauschildt, P. H. 1998, A\&A, 337, 403 (BCAH)

Beckwith, S. V. W., Sargent, A. I., Chini, R. S., \& Guesten, R. 1990, AJ, 99, 924

Bertout, C., Robichon, N., \& Arenou, F. 1999, A\&A, 352, 574

Biver, N., Bockelée-Morvan, D., Colom, P., et al. 1999, Earth, Moon \& Planets, 78, 5

Bouvier, J., Bertout, C., Benz, W., \& Mayor, M. 1986, A\&A, 165, 110

Cernicharo, J., \& Guélin, M. 1987, A\&A, 176, 292

Chiang, E. I., \& Goldreich, P. 1997, ApJ, 490, 368

Chini, R., Kruegel, E., Kreysa, E., Shustov, B., \& Tutukov, A. 1991, A\&A, 252, 220

d'Antona, F., \& Mazzitelli, I. 1997, Mem. Soc. Astron. It., 68, 807 (DM97)

Dartois, E., Dutrey, A., \& Guilloteau, S. 2003, A\&A, 399, 773

Dutrey, A., Guilloteau, S., \& Simon M., 1994, A\&A, 286, 149 (D94)

Dutrey, A., Guilloteau, S., Duvert, G., et al. 1996, A\&A, 309, 493

Dutrey, A., Guilloteau, S., Prato, L., et al. 1998, A\&A, 338, L63

Duvert, G., Guilloteau, S., Ménard, F., Simon, M., \& Dutrey, A. 2000, A\&A, 355, 165

Favata, F., Micela, G., Sciortino, S., \& D’Antona, F. 1998, A\&A, 335, 218

Goldsmith, P. F., Young, J. S., \& Langer, W. D. 1983, ApJS, 51, 203

Gullbring, E., Hartmann, L., Briceno, C., \& Calvet, N. 1998, ApJ, 492, 323

Gullbring, E., et al. 2000, ApJ, 544, 927

Guilloteau, S., \& Dutrey, A., A\&A, 339, 467

Hartmann, L., Hewett, R., Stahler, S., \& Mathieu, R. D. 1986, ApJ, 309,275

Hartmann, L., et al. 1998, ApJ, 495, 385

Herbig, G. H., \& Bell, K. R. 1988, Lick Obs. Bull. No. 1111

Holland, W. S., Greaves, J. S., Zuckerman, B., et al. 1998, Nature, 392, 788

Kenyon, S. J., Dobryzycka, D., \& Hartmann, L. 1994, AJ, 108, 1872

Koerner, D. W., Sargent, A. I., \& Beckwith, S. V. W. 1993, Icarus, 106, 2 (K93)

Lagrange, A. M., Vidal-Madjar, A., Deleuil, M., et al. 1995, A\&A, 296, 499

Mannings, V., Koerner, D. W., \& Sargent, A. I. 1997, Nature, 388, 555

Mouillet, D., Lagrange, A.-M., Beuzit, J.-L., \& Renaud, N. 1997, A\&A, 324, 1083

Osterloh, \& Beckwith, S. V. W. 1995, A\&A

Palla, F., \& Stahler, S. W. 1999, ApJ, 525, 772 (PS99)

Pietu, V., Dutrey, A., \& Kahane, C. 2003, A\&A, 398, 565

Pringle, J. E. 1981, ARA\&A, 19, 137

Schneider, G., Smith, B. A., Becklin, E. E., et al. 1999, ApJ, 513, L127

Simon, M., Dutrey, A., \& Guilloteau, S. 2000, ApJ, 545, 1034

Siess, L., Dufour, E., \& Forestini, M. 2000 A\&A, 358, 593 (SDF)

Vidal-Madjar, A., Lecavelier des Etangs, A., \& Ferlet, R. 1998, Planet. Space Sci., 46, 629 